\title{
Existence of nonoscillatory solutions of nonlinear neutral differential equation of second order
}

\author{
Hussain Ali Mohamad ${ }^{\mathrm{a}, *}$, Bashar Ahmed Jawad ${ }^{\mathrm{b}}$ \\ ${ }^{a}$ Department of mathematics, College of science for women, University of Baghdad, Iraq. \\ ${ }^{b}$ Department of Mathematics, Faculty of Computer Science and Mathematics, University of Kufa, Iraq.
}

\begin{abstract}
In this paper, some necessary and sufficient conditions have been obtained to ensure the existence of nonoscillatory solutions which are bounded below and above by bounded functions. These conditions are more applicable than some known results in the references. An example is included to illustrate the results obtained.
\end{abstract}

Keywords: Existence of positive solution, neutral differential equations, asymptotic behavior.

2010 MSC: 34C10, 34K11.

(C)2019 All rights reserved.

\section{Introduction}

This paper is concerned with the existence of a positive solution of the neutral differential equations of the form

$$
\frac{d^{2}}{d t^{2}}(x(t)-a(t) x(t-\tau))-p(t) f\left(t, x(t), x(t-\sigma), x^{\prime}(t), x^{\prime}(t-\sigma)\right)=0,
$$

where $t \geqslant t_{0}, t_{0} \in \mathbb{R}, \tau>0, \sigma \geqslant 0, a, p \in C\left(\left[t_{0}, \infty\right) ; \mathbb{R}\right), f \in C\left(\left[t_{0}, \infty\right) \times \mathbb{R}^{4} ; \mathbb{R}\right), f$ is bounded function, and $x(t) f\left(t, x(t), x(t-\sigma), x^{\prime}(t), x^{\prime}(t-\sigma)\right)>0, x \neq 0$. By a solution of (1.1) we mean a function $x \in$ $C\left[\left(t_{1}-m, \infty\right), \mathbb{R}\right], m=\max \{\tau, \sigma\}$, for some $t_{1} \geqslant t_{0}$, such that $x(t)+a(t) x(t-\tau)$ is two times continuously differentiable on $\left[t_{1}, \infty\right)$, and $x(t)$ satisfies (1.1) for $t \geqslant t_{1}$. The problem of the existence of the solutions of neutral differential equations has been studied by several authors in the recent years. For related results we refer the reader to $[10,11,12,8,15,16]$, and the references cited therein. Most authors have discussed the existence of a bounded solutions by constant, however there are a few authors who have discussed and gave a conception which guarantees the existence of positive solutions of second order neutral differential equation which are bounded below and above by positive functions. Tanaka [13]

\footnotetext{
*Corresponding author

Email addresses: hussainam_math@csw.uobaghdad.edu.iq (Hussain Ali Mohamad), bashara.hamod@uokufa.edu.iq (Bashar Ahmed Jawad)
}

doi: 10.22436/jmcs.019.01.01

Received: 2018-07-23 Revised: 2018-11-17 Accepted: 2018-11-22 
obtained sufficient conditions for the existence of positive solutions of higher order nonlinear neutral differential equations. Weiming et al. [14] studied existence of nonoscillatory solution of second order neutral differential equations. Culákov et al. [2] studied the existence of nonoscillatory solutions of second order nonlinear neutral differential equations. Olach et al. [6] obtained some sufficient conditions for the existence of a positive solution which is bounded with exponential functions. Olach et al. [5] and [3] obtained some sufficient conditions for the existence of positive solutions which are bounded below and above by positive functions for the nonlinear neutral differential equations of higher order. Olach et al. [4] studied the existence of uncountably many positive solutions which are bounded below and above by positive functions for the first-order nonlinear neutral differential equations. Candan [1] in his paper presented the existence of positive periodic solutions for first order neutral differential equation with distributed deviating arguments. They applied Krasnoselskii's fixed point theorem to obtain the results of existence of bounded solution $x(t) \in[m, M]$. In this paper some necessary and sufficient conditions are established to guarantee the existence of positive solution of (1.1) which is bounded below and bounded above by bounded nonnegative functions, however these conditions are more applicable than the conditions obtained in Theorem 2.1 [6], [5] and [3]. An example is given to illustrate the results obtained. The following lemma and theorem will be used to prove the main results in the next section.

Lemma 1.1 ([7], Krasnoselskii's fixed point theorem). Let $\mathrm{X}$ be a Banach space, $\Omega$ be a bounded closed convex subset of $X$, and let $S_{1}, S_{2}$ be maps of $\Omega$ into $X$ such that $S_{1} x+S_{2} y$ for every pair $x, y \in \Omega$. If $S_{1}$ is contractive and $S_{2}$ is completely continuous, then the equation $S_{1} x+S_{2} x=x$ has a solution in $\Omega$.

Theorem 1.2 ([9], Lebesgue's dominated convergence theorem). Let $f_{\mathrm{n}}$ be a sequence of functions such that $\lim _{n \rightarrow \infty} f_{n}(x)=f(x)$ almost everywhere in $A$ and such that for every $n=1,2,3, \ldots$

$$
\left|f_{n}(x)\right| \leqslant g(x) \text { almost everywhere in } A,
$$

where $g$ is integrable on $A$. Then $\lim _{n \rightarrow \infty} \int f_{n}(x) d x=\int f(x) d x$.

\section{The existence of positive solution}

In this section the existence of positive solution for (1.1) is investigated. In the following results, some necessary and sufficient conditions are obtained to ensure the existence of positive solution for (1.1), which is bounded by two positive functions.

Theorem 2.1. Suppose $p(t) \geqslant 0$ and there exist bounded functions $u, v \in C^{1}\left(\left[t_{0}, \infty\right),[0, \infty)\right)$, a constant $\delta>0$ such that

$$
\begin{gathered}
\frac{u(t)}{u(t-\tau)+\delta} \leqslant a(t) \leqslant \frac{v(t)}{v(t-\tau)}, \quad a(t)<1, \quad v(t-\tau)>0, \quad t \geqslant t_{1} \geqslant t_{0}+m, \\
u(t) \leqslant v(t), \quad t \geqslant t_{0},
\end{gathered}
$$

and there exists $k \in\left(t, t_{1}\right)$ for $t \in\left[t_{0}, t_{1}\right]$ such that

$$
\begin{gathered}
\left(v(t)-v\left(t_{1}\right)\right)\left(1-\frac{u^{\prime}(k)}{v^{\prime}(k)}\right) \geqslant 0, \\
\int_{t_{0}}^{\infty} \int_{s}^{\infty} p(\xi) d \xi d s<\infty, \quad s \geqslant t_{0} .
\end{gathered}
$$

Then (1.1) has a positive solution which is bounded by the functions $u$ and $v$.

Proof. Let $\mathrm{C}\left(\left[\mathrm{t}_{0}, \infty\right), \mathbb{R}\right)$ be the set of all continuous bounded functions with the norm $\|x\|=\sup _{\mathrm{t} \geqslant \mathrm{t}_{0}}|x(\mathrm{t})|$. Then $\mathrm{C}\left(\left[\mathrm{t}_{0}, \infty\right), \mathbb{R}\right)$ is a Banach space. We define a closed, bounded, and convex subset $\Omega$ of $\mathrm{C}\left(\left[\mathrm{t}_{0}, \infty\right), \mathbb{R}\right)$ as follows

$$
\Omega=\left\{x(t) \in C\left(\left[t_{0}, \infty\right), \mathbb{R}\right): u(t) \leqslant x(t) \leqslant v(t), \quad t \geqslant t_{0}\right\}
$$


By (2.4) it follows that

$$
\lim _{t \rightarrow \infty} \int_{t}^{\infty} \int_{s}^{\infty} p(\xi) d \xi d s=0, \quad s \geqslant t
$$

It follows that for every $\varepsilon>0$, there exists $t_{1} \geqslant t_{0}$ such that

$$
\int_{t}^{\infty} \int_{s}^{\infty} p(\xi) d \xi d s<\varepsilon, \quad s \geqslant t \geqslant t_{1} .
$$

For simplicity let $f(t, x(t))=f\left(t, x(t), x(t-\sigma), x^{\prime}(t), x^{\prime}(t-\sigma)\right)$, we now define maps $S_{1}, S_{2} \in C\left(\left[t_{0}, \infty\right), \mathbb{R}\right)$ as follows

$$
\begin{aligned}
& \left(S_{1} x\right)(t)= \begin{cases}\left(S_{1} x\right)\left(t_{1}\right), & t_{0} \leqslant t \leqslant t_{1}, \\
a(t) x(t-\tau), & t \geqslant t_{1},\end{cases} \\
& \left(S_{2} x\right)(t)= \begin{cases}\left(S_{2} x\right)\left(t_{1}\right)+v(t)-v\left(t_{1}\right), & t_{0} \leqslant t \leqslant t_{1}, \\
-\int_{t}^{\infty} \int_{s}^{\infty} p(\xi) f(\xi, x(\xi)) d \xi d s, & t \geqslant t_{1} .\end{cases}
\end{aligned}
$$

We will show that for any $x, y \in \Omega$ we have $S_{1} x+S_{2} y \in \Omega$. Let $x, y \in \Omega$ and $t \geqslant t_{1}$ we obtain

$$
\begin{aligned}
\left(S_{1} x\right)(t)+\left(S_{2} y\right)(t) & =a(t) x(t-\tau)-\int_{t}^{\infty} \int_{s}^{\infty} p(\xi) f(\xi, x(\xi)) d \xi d s \\
& \leqslant a(t) v(t-\tau) \\
& \leqslant v(t) .
\end{aligned}
$$

For $\mathrm{t} \in\left[\mathrm{t}_{0}, \mathrm{t}_{1}\right]$, we have

$$
\begin{aligned}
\left(S_{1} x\right)(t)+\left(S_{2} y\right)(t) & =\left(S_{1} x\right)\left(t_{1}\right)+\left(S_{2} y\right)\left(t_{1}\right)+v(t)-v\left(t_{1}\right) \\
& \leqslant v\left(t_{1}\right)+v(t)-v\left(t_{1}\right) \\
& =v(t)
\end{aligned}
$$

Since $f(t, x(t))$ is bounded so we get

$$
\lim _{t \rightarrow \infty} \int_{t}^{\infty} \int_{s}^{\infty} p(\xi) f(\xi, x(\xi)) d \xi d s=0, \quad s \geqslant t .
$$

Hence, for $t \geqslant t_{1}$ we get

$$
\begin{aligned}
\left(S_{1} x\right)(t)+\left(S_{2} y\right)(t) & =a(t) x(t-\tau)-\int_{t}^{\infty} \int_{s}^{\infty} p(\xi) f(\xi, x(\xi)) d \xi d s \\
& >a(t) u(t-\tau)-(1-a(t)) \varepsilon) \\
& \geqslant a(t)(u(t-\tau)+\varepsilon),
\end{aligned}
$$

by choosing $\delta=\varepsilon$, and using condition (2.1) it follows from (2.5)

$$
\left(S_{1} x\right)(t)+\left(S_{2} y\right)(t) \geqslant u(t), \quad t \geqslant t_{1} .
$$

Let $t \in\left[t_{0}, t_{1}\right]$, then by Cauchy mean value theorem in virtue of $(2.3)$, for $t \in\left[t_{0}, t_{1}\right]$ there exists $k \in\left(t, t_{1}\right)$ such that

$$
\begin{aligned}
& u^{\prime}(k)\left(v\left(t_{1}\right)-v(t)\right)=v^{\prime}(k)\left(u\left(t_{1}\right)-u(t)\right), \\
& v^{\prime}(k) u(t)=v^{\prime}(k) u\left(t_{1}\right)+u^{\prime}(k)\left(v(t)-v\left(t_{1}\right)\right), \\
& u(t)=u\left(t_{1}\right)+\frac{u^{\prime}(k)}{v^{\prime}(k)}\left(v(t)-v\left(t_{1}\right)\right), \\
& u(t) \leqslant u\left(t_{1}\right)+v(t)-v\left(t_{1}\right), \quad t_{0} \geqslant t \geqslant t_{1} .
\end{aligned}
$$


Then for $t \in\left[t_{0}, t_{1}\right]$ and for any $x, y \in \Omega$, we obtain

$$
\begin{aligned}
\left(S_{1} x\right)(t)+\left(S_{2} y\right)(t) & =\left(S_{1} x\right)\left(t_{1}\right)+\left(S_{2} y\right)\left(t_{1}\right)+v(t)-v\left(t_{1}\right) \\
& \geqslant u\left(t_{1}\right)+v(t)-v\left(t_{1}\right) \geqslant u(t) .
\end{aligned}
$$

Thus we have proved that $S_{1} x+S_{2} y \in \Omega$ for any $x, y \in \Omega$. We will show that $S_{1}$ is a contraction mapping on $\Omega$. For $x, y \in \Omega$ and $t \geqslant t_{1}$ we have

$$
\begin{aligned}
\left\|S_{1} x-S_{1} y\right\| & =\sup _{t \geqslant t_{1}}|a(t) x(t-\tau)-a(t) y(t-\tau)| \\
& =\sup _{t \geqslant t_{1}} a(t)|x(t-\tau)-y(t-\tau)| \\
& \left.\leqslant c_{1} \sup _{t \geqslant t_{1}}|x(t-\tau)-y(t-\tau)|, \quad \text { (where } a(t) \leqslant c_{1}<1\right) \\
& =c_{1}\|x-y\| .
\end{aligned}
$$

Also for $t \in\left[t_{0}, t_{1}\right]$, we get

$$
\begin{aligned}
\left\|S_{1} x-S_{1} y\right\| & =\sup _{t_{0} \leqslant t \leqslant t_{1}}\left|\left(S_{1} x\right)(t)-\left(S_{1} y\right)(t)\right| \\
& =\left|a\left(t_{1}\right) x\left(t_{1}-\tau\right)-a\left(t_{1}\right) y\left(t_{1}-\tau\right)\right| \\
& =a\left(t_{1}\right)\left|x\left(t_{1}-\tau\right)-y\left(t_{1}-\tau\right)\right| \\
& \leqslant a\left(t_{1}\right) \sup _{t_{0} \leqslant t \leqslant t_{1}}|x(t-\tau)-y(t-\tau)| \\
& =a\left(t_{1}\right)\|x-y\| .
\end{aligned}
$$

We conclude that $S_{1}$ is a contraction mapping on $\Omega$. We now show that $S_{2}$ is completely continuous. First we will show that $S_{2}$ is continuous. Let $x_{k}=x_{k}(t) \in \Omega$ be such that $\lim _{k \rightarrow \infty} x_{k}(t)=x(t)$. Since $\Omega$ is closed, we conclude that $x(t) \in \Omega$. For $t \geqslant t_{1}$ we have

$$
\begin{aligned}
\left\|\left(S_{2} x_{k}\right)(t)-\left(S_{2} x\right)(t)\right\| & =\sup _{t \geqslant t_{1}}\left|\left(S_{2} x_{k}\right)(t)-\left(S_{2} x\right)(t)\right| \\
& \leqslant \mid \int_{t}^{\infty} \int_{s}^{\infty} p(\xi)\left(f\left(\xi, \mathbf{x}_{\mathbf{k}}(\xi)\right)-f(\xi, \mathbf{x}(\xi)) d \xi d s \mid\right. \\
& \leqslant \int_{t}^{\infty} \int_{s}^{\infty}\left|p(\xi)\left(f\left(\xi, \mathbf{x}_{\mathbf{k}}(\xi)\right)-f(\xi, \mathbf{x}(\xi))\right) d \xi d s\right|
\end{aligned}
$$

According to (2.5), we get

$$
\int_{t_{1}}^{\infty} \int_{s}^{\infty} p(\xi) f\left(\xi, x(\xi), x(\xi-\sigma), x^{\prime}(\xi), x^{\prime}(\xi-\sigma)\right) d \xi d s<\infty .
$$

Since

$$
\lim _{k \rightarrow \infty} f\left(t, x_{k}(t)\right)=f(t, x(t)),
$$

then by applying the Lebesgue dominated convergence Theorem 1.2, we obtain

$$
\lim _{k \rightarrow \infty}\left\|\left(S_{2} x_{k}\right)(t)-\left(S_{2} x\right)(t)\right\|=0 .
$$

This means that $S_{2}$ is continuous. We now show that $S_{2} \Omega$ is relatively compact. It is sufficient to show by Arzelá-Ascoli theorem that the family of functions $\left\{S_{2} x: x \in \Omega\right\}$ is uniformly bounded and equicontinuous on $\left[t_{0}, \infty\right]$. The uniform boundedness follows from the definition of $\Omega$. For the equicontinuity we only need to show that for any given $\varepsilon>0$ the interval $\left[t_{0}, \infty\right]$ can be decomposed into finite subintervals 
in such a way that on each subinterval all functions of the family have a change of amplitude less than $\varepsilon$. Then with regard to (2.6), for $x \in \Omega$ and any $\varepsilon>0$, we take $t_{*} \geqslant t_{1}$ large enough so that

$$
\int_{t_{*}}^{\infty} \int_{s}^{\infty} p(\xi) f\left(\xi, x(\xi), x(\xi-\sigma), x^{\prime}(\xi), x^{\prime}(\xi-\sigma)\right) d \xi d s<\frac{\varepsilon}{2}
$$

Then, for $x \in \Omega, t_{*} \leqslant T_{1}<T_{2}$, we have

$$
\begin{aligned}
\left\|\left(S_{2} x\right)\left(T_{2}\right)-\left(S_{2} x\right)\left(T_{1}\right)\right\| \leqslant & \mid-\int_{T_{2}}^{\infty} \int_{s}^{\infty} p(\xi) f(\xi, x(\xi)) d \xi d s \\
& +\int_{T_{1}}^{\infty} \int_{s}^{\infty} p(\xi) f(\xi, x(\xi)) d \xi d s \mid \\
\leqslant & \int_{T_{2}}^{\infty} \int_{s}^{\infty} p(\xi) f(\xi, x(\xi)) d \xi d s \\
& +\int_{T_{1}}^{\infty} \int_{s}^{\infty} p(\xi) f(\xi, x(\xi)) d \xi d s,
\end{aligned}
$$

hence we get

$$
\left\|\left(S_{2} x\right)\left(T_{2}\right)-\left(S_{2} x\right)\left(T_{1}\right)\right\|<\frac{\varepsilon}{2}+\frac{\varepsilon}{2}=\varepsilon .
$$

For $x \in \Omega$ and $t_{1} \leqslant T_{1}<T_{2} \leqslant t_{*}$, it yields

$$
\begin{aligned}
\left|\left(S_{2} x_{k}\right)\left(T_{2}\right)-\left(S_{2} x\right)\left(T_{1}\right)\right| \leqslant & 1-\int_{T_{2}}^{\infty} \int_{s}^{\infty} p(\xi) f(\xi, x(\xi)) d \xi d s \\
& +\int_{T_{1}}^{\infty} \int_{s}^{\infty} p(\xi) f(\xi, x(\xi)) d \xi d s \mid \\
\leqslant & \int_{T_{1}}^{T_{2}} \int_{s_{1}}^{\infty} p(\xi) f\left(\xi, x(\xi), x(\xi-\sigma), x^{\prime}(\xi), x^{\prime}(\xi-\sigma)\right) d \xi d s \\
\leqslant & \max _{t_{1} \leqslant s \leqslant t_{*}}\left\{\int_{s}^{\infty} p(\xi) f\left(\xi, x(\xi), x(\xi-\sigma), x^{\prime}(\xi), x^{\prime}(\xi-\sigma)\right) d \xi\right\}\left(T_{2}-T_{1}\right) .
\end{aligned}
$$

Thus there exists $\delta_{1}=\frac{\varepsilon}{M}$, where $M=\max _{t_{1} \leqslant s \leqslant t_{*}}\left\{\int_{s}^{\infty} p(\xi) f\left(\xi, \chi(\xi), \chi(\xi-\sigma), \chi^{\prime}(\xi), \chi^{\prime}(\xi-\sigma)\right) d \xi\right\}$ such that

$$
\mid\left(S_{2} \times\left(T_{2}\right)-\left(S_{2} \times\left(T_{1}\right) \mid<\varepsilon, \quad \text { if } 0<T_{2}-T_{1}<\delta_{1} .\right.\right.
$$

Finally for any $x \in \Omega, t_{0} \leqslant T_{1}<T_{2} \leqslant t_{1}$, there exists $k_{1} \in\left(T_{1}, T_{2}\right)$ and $\delta_{2}=\frac{\varepsilon}{\left|v^{\prime}\left(k_{1}\right)\right|}>0$ such that

$$
\begin{aligned}
\left|\left(S_{2} x\right)\left(T_{2}\right)-\left(S_{2} x\right)\left(T_{1}\right)\right| & =\left|v\left(T_{2}\right)-v\left(T_{1}\right)\right| \\
& =\left|v^{\prime}\left(k_{1}\right)\right|\left(T_{2}-T_{1}\right)<\varepsilon, \quad \text { if } 0<T_{2}-T_{1}<\delta_{2} .
\end{aligned}
$$

Then $\left\{S_{2} x: x \in \Omega\right\}$ is uniformly bounded and equicontinuous on $\left[t_{0}, \infty\right)$, and hence $S_{2} \Omega$ is relatively compact subset of $C\left(\left[t_{0}, \infty\right), \mathbb{R}\right)$. By Lemma 1.1 there is an $x_{0} \in \Omega$ such that $S_{1} x_{0}+S_{2} x_{0}=x_{0}$. We conclude that $x_{0}(t)$ is a positive solution of (1.1). The proof is complete.

Corollary 2.2. Suppose that there exist bounded functions $u, v \in \mathrm{C}^{1}\left(\left[\mathrm{t}_{0}, \infty\right),[0, \infty)\right)$, a constant $\delta>0$ and a positive integer $n$ such that

$$
\begin{gathered}
\frac{u(t)}{u(t-\tau)+\delta}+n-1 \leqslant n a(t) \leqslant \frac{v(t)}{v(t-\tau)}+(n-1) a(t), \quad a(t)<1, \\
u(t)>0, \quad v(t)>0, \quad t \geqslant t_{1} \geqslant t_{0}+m,
\end{gathered}
$$

in addition to the conditions (2.2), (2.3), (2.4) hold. Then (1.1) has a positive solution which is bounded by the functions $\mathrm{u}$ and $v$.

Proof. We claim that condition (2.7) implies condition (2.1), we will use the induction to show it when 
$n=1$ there is nothing to prove, when $n=2$ then it follows from condition (2.7)

$$
\begin{aligned}
\frac{u(t)}{u(t-\tau)+\delta}+1 & \leqslant 2 a(t) \leqslant \frac{v(t)}{v(t-\tau)}+a(t), \\
\frac{u(t)}{u(t-\tau)+\delta} & \leqslant \frac{1}{2}\left(\frac{u(t)}{u(t-\tau)+\delta}+a(t)\right) \\
& \leqslant \frac{1}{2}\left(\frac{u(t)}{u(t-\tau)+\delta}+1\right) \\
& \leqslant a(t) \\
& \leqslant \frac{1}{2}\left(\frac{v(t)}{v(t-\tau)}+a(t)\right) \\
& \leqslant \frac{v(t)}{v(t-\tau)},
\end{aligned}
$$

we can treat similarly to show it when $n=3,4, \cdots$. Hence all conditions of Theorem 2.1 hold, then according to Theorem 2.1, equation (1.1) has a positive solution which is bounded by the functions $u$ and $v$.

Example 2.3. Consider the following non-linear neutral differential equation

$$
\frac{d^{2}}{d t^{2}}(x(t)-a(t) x(t-1))+p(t)\left(e^{-t}+2 e^{-t+\frac{3}{2}}\right)=0, \quad t \geqslant \frac{1}{2},
$$

where $\frac{1}{4} \leqslant a(t)<1, p(t)=\frac{9}{4} e^{-\frac{t}{2}}, f(t, x(t))=e^{-t}+2 e^{-t+\frac{3}{2}}$.

Solution: Let $u(t)=e^{-4}+e^{-2 t}$ and $v(t)=\frac{3}{2}-e^{-t}, t \geqslant \frac{1}{2}$. The condition (2.2) clearly satisfies, now we will show that the condition (2.1) holds, choose $\delta=\frac{1}{2}$ then it follows that

$$
\frac{e^{-4}+e^{-2 t}}{e^{-4}+e^{-2(t-1)}+\frac{1}{2}}<a(t)<\frac{\frac{3}{2}-e^{-t}}{\frac{3}{2}-e^{-(t-1)}}, \text { for all } t \geqslant \frac{3}{2} .
$$

To see condition (2.3) consider that

$$
\left(\frac{3}{2}-e^{-t}-\left(\frac{3}{2}-e^{-1}\right)\right)\left(1-\frac{-2 e^{-2 k}}{e^{-k}}\right) \geqslant \frac{1}{e}\left(1+2 e^{-k}\right)>0, \text { for all } k .
$$

It remains to show that condition (2.4) holds

$$
\int_{\mathrm{t}_{0}}^{\infty} \int_{\mathrm{s}}^{\infty} \mathrm{p}(\mathrm{t}) \mathrm{dt} \mathrm{ds}=\int_{\frac{1}{2}}^{\infty} \int_{s}^{\infty} \frac{9}{4} e^{-\frac{\mathrm{t}}{2}} \mathrm{dt} \mathrm{ds}=9 \mathrm{e}^{-\frac{1}{4}}
$$

Then all conditions of Theorem 2.1 (or Corollary 2.2) are satisfies. According to Theorem 2.1 (or Corollary 2.2), equation (2.8) has a positive solution which is bounded by the functions $u$ and $v$.

Theorem 2.4. Suppose $\mathrm{p}(\mathrm{t}) \geqslant 0$ and there exist bounded functions $\mathrm{u}, v \in \mathrm{C}^{1}\left(\left[\mathrm{t}_{0}, \infty\right),[0, \infty)\right)$, a constant $\mathrm{c}>0$ such that

$$
\begin{gathered}
u(t) \leqslant v(t), \quad t \geqslant t_{0}, \\
v(t-\tau)-v\left(t_{1}-\tau\right)-u(t-\tau)+u\left(t_{1}-\tau\right) \geqslant 0, \quad t \in\left[t_{0}, t_{1}\right], \\
\frac{1}{u(t-\tau)}\left(\int_{t}^{\infty} \int_{s}^{\infty} p(\xi) f\left(\xi, v(\xi), v(\xi-\tau), v^{\prime}(s), v^{\prime}(\xi-\tau)\right) d \xi d s\right) \\
\leqslant a(t)-1 \\
\leqslant \frac{1}{v(t-\tau)}\left(\int_{t}^{\infty} \int_{s}^{\infty} p(\xi) f\left(\xi, u(\xi), u(\xi-\tau), u^{\prime}(s), u^{\prime}(\xi-\tau)\right) d \xi d s\right) \\
\leqslant c<1, \quad t \geqslant t_{1} .
\end{gathered}
$$

Then (1.1) has a positive solution which is bounded by the functions $u$ and $v$. 
Proof. The proof is similar to the proof of Theorem 2.1 and will be omitted.

Corollary 2.5. Suppose that there exist bounded functions $u, v \in \mathrm{C}^{1}\left(\left[\mathrm{t}_{0}, \infty\right),[0, \infty)\right)$, a constant $\mathrm{c}>0$ such that (2.9), (2.11) hold in addition to the condition

$$
v^{\prime}(t-\tau) \leqslant u^{\prime}(t-\tau), \quad t \in\left[t_{0}, t_{1}\right]
$$

Then (1.1) has a positive solution which is bounded by the functions $u$ and $v$.

Proof. We only need to prove that condition (2.12) implies (2.10). Let $t \in\left[t_{0}, t_{1}\right]$ and set

$$
H(t)=v(t-\tau)-v\left(t_{1}-\tau\right)-u(t-\tau)+u\left(t_{1}-\tau\right) .
$$

Then with regard to (2.12), it follows that

$$
H^{\prime}(t)=v^{\prime}(t-\tau)-u^{\prime}(t-\tau)
$$

Since $H\left(t_{1}\right)=0$ and $H^{\prime}(t) \leqslant 0$ for $t \in\left[t_{0}, t_{1}\right]$, this implies that

$$
H(t)=v(t-\tau)-v\left(t_{1}-\tau\right)-u(t-\tau)+u\left(t_{1}-\tau\right) \geqslant 0, \quad t_{0} \leqslant t \leqslant t_{1} .
$$

Hence all conditions of Theorem 2.4 are satisfied.

Corollary 2.6. Suppose that there exists a function $v \in \mathrm{C}^{1}\left(\left[\mathrm{t}_{0}, \infty\right),(0, \infty)\right)$, a constant $\mathrm{c}>0$ and $\mathrm{t}_{1} \geqslant \mathrm{t}_{0}+\mathrm{m}$ such that

$$
\left.a(t)=1+\frac{1}{v(t-\tau)} \int_{t}^{\infty} p(s) f\left(s, v(s), v(s-\tau), v^{\prime}(s), v^{\prime}(s-\tau)\right) d s\right) \leqslant \delta<1, \quad t \geqslant t_{1} .
$$

Then (1.1) has a solution $x(t)=v(t-\tau), t \geqslant t_{1}$.

Proof. We put $u(t-\tau)=v(t-\tau)$ and apply Theorem 2.4.

\section{Conclusion}

This paper is concerned with establishing some sufficient conditions to ensure the existence of a positive solution which is bounded by two bounded functions. Two main results were obtained with corollaries.

\section{References}

[1] T. Candan, Existence of positive periodic solutions of first-order neutral differential equations, Math. Methods Appl. Sci., 40 (2017), 205-209. 1

[2] I. Culákov, L. Hanutiaková, R. Olach, Existence for positive solutions of second-order neutral nonlinear differential equations, Appl. Math. Lett., 22 (2009), 1007-1010. 1

[3] B. Dorociaková, M. Kubjatková, R. Olach, Existence of Positive Solutions of Neutral Differential Equations, Abstr. Appl. Anal., 2012 (2012), 14 pages. 1

[4] B. Dorociaková, M. Kubjatková, R. Olach, Uncountably many solutions of first-order neutral nonlinear differential equations, Adv. Difference Equ., 2013 (2013), 8 pages. 1

[5] B. Dorociaková, A. Najmanová, R. Olach, Existence of Nonoscillatory Solutions of First-Order Neutral Differential Equations, Abstr. Appl. Anal., 2011 (2011), 9 pages. 1

[6] B. Dorociaková, R. Olach, Existence of Positive Solutions of Delay Differential Equations, Tatra Mt. Math. Publ., 43 (2009), 63-70. 1

[7] L. H. Erbe, Q. Kong, B. G. Zhang, Oscillation Theory for Functional Differential Equations, Marcel Dekker, New York, (1995). 1.1

[8] E. K. Essel, E. Yankson, On The Existence of Positive Periodic Solutions for Totally Nonlinear Neutral Differential Equations of The Second-Order With Functional Delay, Opuscula Math., 34 (2014), 469-481. 1

[9] I. Györi, G. Ladas, Oscillation Theory of Delay Differential Equations, The Clarendon Press, Oxford University Press, New York, (1991). 1.2 
[10] F. Kong, Existence of non-oscillatory solutions of a kind of first-order neutral differential equation, Math. Commun., 22 (2017), 151-164. 1

[11] H. A. Mohamad, I. Z. Mushtt, Oscillation of Second Order Nonlinear Neutral Differential Equations, Pure Appl. Math. J., 4 (2015), 62-65. 1

[12] Ö, Öcalan, Existence of positive solutions for a neutral differential equation with positive and negative coefficients, Appl. Math. Lett., 22 (2009), 84-90. 1

[13] S. Tanaka, Existence of Positive Solutions of Higher Order Nonlinear Neutral Differential Equations, Rocky Mountain J. Math., 30 (2000), 1139-1149. 1

[14] G. Weiming, C. Jinfa, C. Yuming, Existence of Nonoscillatory Solution of Second Order Neutral Differential Equation, Bull. Inst. Math. Acad. Sin. (N.S.), 2 (2007), 785-795. 1

[15] A. J. Yang, Z. G. Zhang, W. G. Ge, Existence of Nonoscillatory Solutions of Second-Order Nonlinear Neutral Differential Equations, Indian J. Pure Appl. Math., 39 (2008), 227-235. 1

[16] Y. H. Yu, H. Z. Wang, Nonoscillatory solutions of second-order nonlinear neutral delay equations, J. Math. Anal. Appl., 311 (2005), 445-456. 1 